\title{
Documenting elimination of co-circulating COVID-19 clusters using genomics in New South Wales, Australia
}

\author{
Alicia Arnott ${ }^{1,2,3 \dagger}$, Jenny Draper ${ }^{1 \dagger}$, Rebecca J. Rockett ${ }^{2,3}$, Connie Lam²,3, Rosemarie Sadsad ${ }^{3,4}$, Mailie Gall ${ }^{1}$, \\ Elena Martinez ${ }^{1,3}$, Roy Byun ${ }^{5}$, Jennie Musto ${ }^{5}$, Ben Marais ${ }^{2,6}$, Sharon C.-A. Chen ${ }^{1,2,3}$, Jen Kok ${ }^{1,2,3}$, \\ Dominic E. Dwyer ${ }^{1,2,3}$ and Vitali Sintchenko $1,2,3^{*}$
}

\begin{abstract}
Objective: To adapt 'fishplots' to describe real-time evolution of SARS-CoV-2 genomic clusters.

Results: This novel analysis adapted the fishplot to depict the size and duration of circulating genomic clusters over time in New South Wales, Australia. It illuminated the effectiveness of interventions on the emergence, spread and eventual elimination of clusters and distilled genomic data into clear information to inform public health action.
\end{abstract}

Keywords: SARS-CoV-2, Genomic epidemiology, Bioinformatics, Whole genome sequencing, Australia

\section{Introduction}

Since the arrival of the first COVID-19 case in Australia, the Pathogen Genomics Team at New South Wales (NSW) Health Pathology's Institute of Clinical Pathology and Medical Research (ICPMR) and the University of Sydney have implemented prospective and responsive whole genome sequencing (WGS) of confirmed SARS-CoV-2 infections. A strong positive correlation between genomics-informed clusters and epidemiologically linked cases was rapidly established, and prospective SARS-CoV-2 WGS transitioned from a novel tool with unproven relevance to an essential element of NSW's COVID-19 public health response [1, 2]. Key to this transformation and the uptake of genomics by public health professionals has been the development and

*Correspondence: Vitali.Sintchenko@health.nsw.gov.au

${ }^{\dagger}$ Alicia Arnott and Jenny Draper contributed equally to this manuscript

${ }^{1}$ Centre for Infectious Diseases and Microbiology Laboratory Services,

NSW Health Pathology - Institute of Clinical Pathology and Medical

Research, Westmead, NSW, Australia

Full list of author information is available at the end of the article testing of novel methods to enable better visualisation and clear communication of genomic results.

In this correspondence we describe the adaptation and application of 'fishplots' to display COVID-19 genomic cluster architecture and evolution over time. The fishplot is a variant of a streamgraph, originally developed to enable visualisation of clonal tumour evolution [3]. We have adapted fishplot analysis to describe SARSCoV-2 virus population dynamics over time, generating an "epi-fishplot" analogous to a stacked epidemiological curve (Fig. 1) [4]. This analysis simultaneously depicts the relative size and duration of circulating SARS-CoV-2 genomic clusters, defined by comparison of SARS-CoV-2 consensus sequences, over the course of the local epidemic. While a conventional phylogenetic tree represents genomic relatedness between individual cases, epi-fishplot analysis enables the timing and impact of key public health interventions to be easily identified and monitored, such as the successful elimination of circulating genomic clusters within the NSW population as a result of effective public health policies (Fig. 1). 


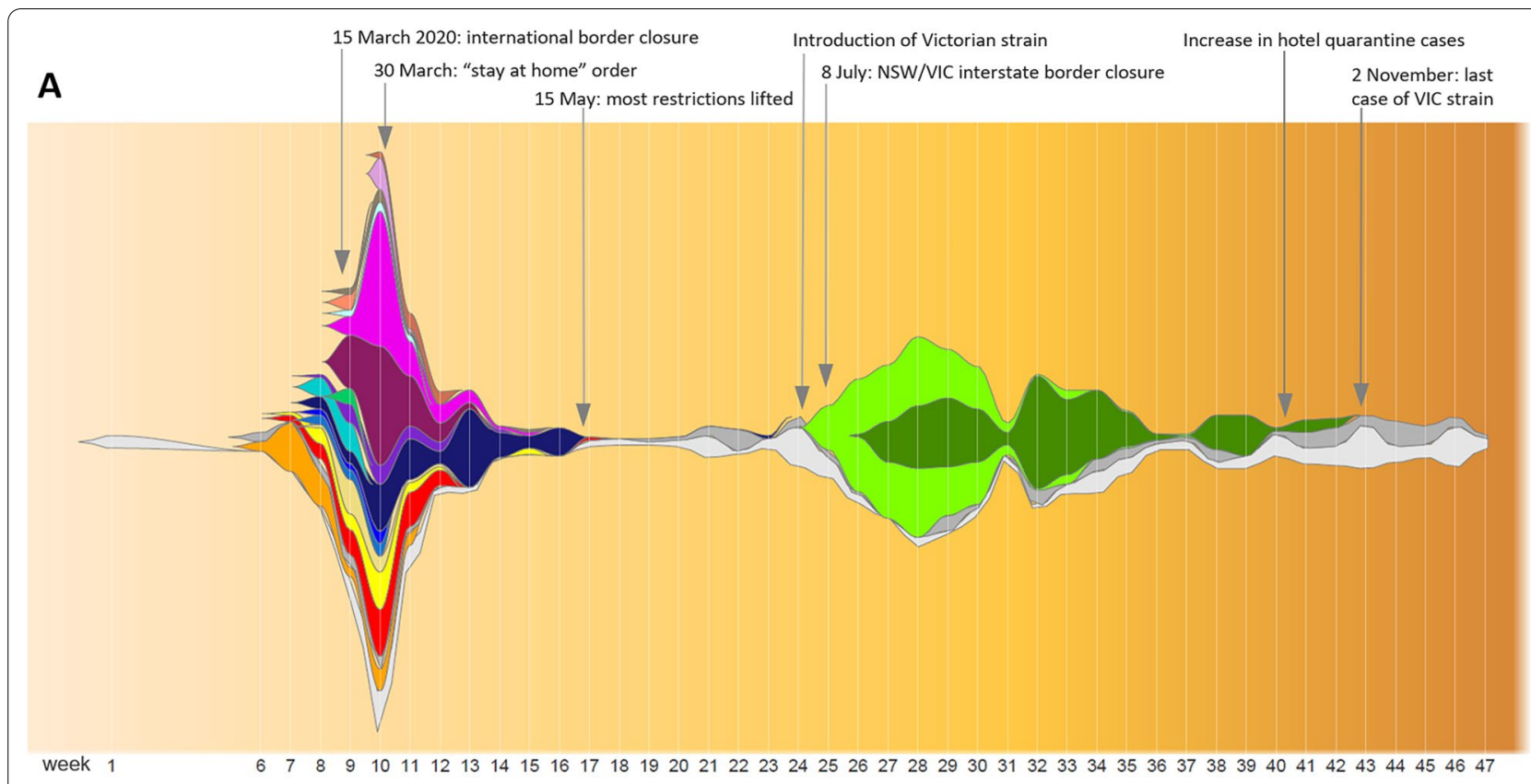

\section{B NSW Cluster}

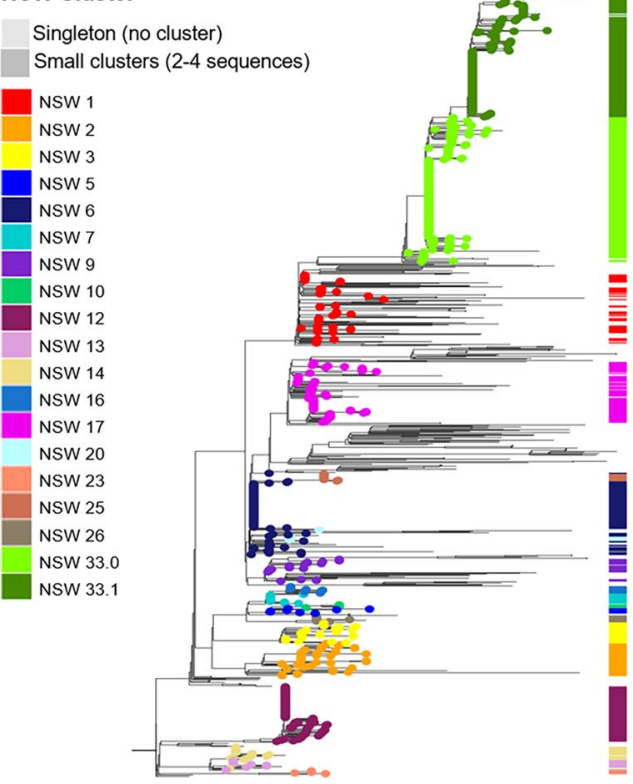

Fig. 1 Epi-fishplot illustrating the rise and fall of SARS-CoV-2 genomic clusters in NSW over the course of the pandemic in 2020 (plotted by epidemiological week, from 22/1/2020). a The height of the plot at each week (vertical white line) is proportional to the total number of genomes obtained from COVID-19 cases sampled that week. The colour represents the assigned genomic cluster. The full height of the Y-axis is proportional to the largest number of sequences obtained for any time point (185 sequences, sampled during the $10^{\text {th }}$ week of 22-28 March 2020). This plot illustrates large number of distinct genomic clusters introduced from overseas to Australia during the initial months of the pandemic, and their rapid elimination as a consequence of border closure and lockdown measures. It also illustrates the spread and steady elimination of a "second-wave" outbreak introduced from the state of Victoria prior to interstate border closure, consisting of two related clusters, NSW33.0 and NSW33.1. b Classic phylogenetic tree representation of the same dataset, representing the relatedness of all sequenced cases in NSW. Clusters with $\geq 5$ sequences are indicated in the metabar and coloured according to the colour scheme of the epi-fishplot (a). The phylogenetic tree was constructed as described previously ${ }^{1}$ 


\section{Main text}

The state of NSW is Australia's most populous with 8 million residents accounting for $31 \%$ of the Australian population, 65\% of whom live in Greater Sydney. Since the Australian border was closed to all non-Australian travellers on March $15^{\text {th }}$ and Australians returning from overseas have been required to complete a mandatory 14-day quarantine in designated hotels since March $28^{\text {th }}$, NSW has accommodated over half of the travellers returning to Australia by air. Despite this and at the time of writing (March $28^{\text {th }}, 2021$ ), NSW accounted for just $17 \%(n=5094)$ of Australia's confirmed COVID-19 cases with no extensive local transmission of any strains linked to foreign travellers returning after March $15^{\text {th }}$. In total, overseas acquired cases restricted to hotel quarantine outweighed locally acquired cases, accounting for 59\% and $41 \%$, respectively. The multidisciplinary public health response to COVID-19 implemented in NSW is co-ordinated by the Public Health Emergency Operations Centre within the NSW Ministry of Health (NSW Health) [5]. Key to terminating local SARS-CoV-2 transmission chains has been the active case finding and contract tracing conducted by NSW Health for each laboratory-confirmed case, which included generating a SARS-CoV-2 genomic sequence to monitor its spread.

The integration of SARS-CoV-2 genomics into routine public health response has addressed key limitations of conventional epidemiological methods including poor or incorrect case recall, and confirmation of contentious or tenuous links. Furthermore, genomics has been instrumental in the timely identification of links between cases for which epidemiological links were not immediately apparent, supplementing conventional contact tracing methods and informing targeted public health resource allocation [1]. In NSW, the integration of genomics into routine public health practice includes prioritisation of clinical samples for rapid sequencing, weekly verbal and written reports to NSW Health and customised on-demand reports for urgent, highpriority cases. As of the time of writing (28th March, 2021), the Pathogen Genomics Team had generated and shared 1144 complete SARS-CoV-2 genomes representing $28 \%$ of all confirmed COVID-19 cases in NSW (Supplementary data: Additional file 1). On the basis of epidemiological information provided by NSW Health and specific single nucleotide polymorphism (SNP) profiles [1], these genomes have been classified into 53 genomic clusters. The median duration, i.e. circulation in the community, of identified genomic clusters was two weeks, although this was highly variable (range: 1-16 weeks) with clusters consisting of a median of three cases (range: 2-204 cases; Fig. 1). Epidemiological data also enabled the Pathogen Genomics Team to report which cases belong to specific transmission chains, the detail of which is described in regular reports that overlay conventional phylogenetic trees with infographics representing the supplied epidemiological data. An epi-fishplot has been generated as part of the report to provide a populationlevel overview of SARS-CoV-2 clusters co-circulating in the local community, illuminating the effectiveness of public health measures on the emergence, spread and eventual elimination of transmission chains within the local population (Fig. 1) [5].

Our data illustrate the two epidemiologically distinct waves of SARS CoV-2 infections experienced in NSW in 2020, the peaks of which occurred in late March and July, respectively. The first wave resulted from multiple independent introductions of genomically distinct viruses by overseas travellers prior to the closure of international borders on March $15^{\text {th }}$. Sustained local transmission of introduced strains was the exception and the median duration in weeks for the 17 first wave clusters identified ( $\geq 5$ cases) was 4 (range: 1-10: Fig. 1). By mid-May 2020, local transmission of all clusters identified in the first wave had been eliminated, despite continued importation of overseas acquired cases into the NSW hotel quarantine system. This state of elimination, without significant or sustained local transmission (no clusters consisting of $\geq 5$ cases) was maintained for the following two months (Fig. 1).

Genome sequencing confirmed that the second wave was seeded by a domestic importation from neighbouring state, Victoria, in early July 2020. The interstate resident travelled to NSW immediately prior to the border between the two states closing for the first time in 100 years in order to prevent spill-over from Victoria into NSW. Confirming that importation was the source of the initial second wave cluster (NSW33.0: Fig. 1), and not undetected community transmission, provided important reassurance that the NSW public health measures in place were effective. The initial infection event occurred at a large licensed venue situated in close proximity to a highway traversing the east coast of Australia, which facilitated infection of multiple individuals and enabled this strain to initiate several transmission chains amongst the local population (Fig. 1). Genome sequencing confirmed that public health measures had eliminated the NSW33.0 cluster by mid-September 2020, and the NSW33.1 subcluster by early November 2020.

Globally, the COVID-19 pandemic has necessitated extraordinary and often innovative public health responses to prevent and control widespread virus transmission. The high frequency of asymptomatic infections or subclinical disease and comparatively limited genomic diversity of circulating strains has exposed limitations of conventional epidemiological and genomic approaches when deployed in isolation to contain the spread of SARS-CoV-2. 
Genomic sequencing has been recognised as a powerful public health tool that provides a unique level of resolution and oversight and addresses critical limitations of conventional epidemiological methods [6]. The epi-fish approach described above can be equally applied to other pathogens of public health importance subjected to integrated prospective genomic surveillance. The successful integration of genomics into routine public health response relies on a strong working partnership between public health practitioners and laboratory professionals, with evidence synthesis and visualisation in order to distil complex genomic data into the information which can guide and benchmark public health actions.

\section{Limitations}

A consensus genome sequence could not be obtained from every confirmed case of COVID-19 detected in NSW during the study period. Sequences were reliably recovered from samples with a diagnostic PCR Ct value $\leq 30$. However, the proportion of sequenced cases (28\%) was high compared to that reported by most countries around the world [7] and more than sufficient to achieve the primary goal of this study which was to demonstrate the application and utility of the Epifish package.

\begin{abstract}
Abbreviations
NSW: New South Wales; WGS: Whole genome sequencing; ICPMR: Institute of Clinical Pathology and Medical Research; SNP: Single nucleotide polymorphism; PCR: Polymerase chain reaction; Ct: PCR cycle threshold.
\end{abstract}

\section{Supplementary Information}

The online version contains supplementary material available at https://doi. org/10.1186/s13104-021-05827-x.

Additional file 1. SARS-CoV-2 genome sequences included in the visualisation of COVID-19 activity in New South Wales, Australia.

\section{Acknowledgements}

We acknowledge the Sydney Informatics Hub and use of the University of Sydney's high-performance computing cluster, Artemis. We thank the NSW Health Pathology partner laboratories, as well as ACT Pathology, Douglass Hanly Moir, Australian Clinical Laboratories and Laverty Pathology for referring samples for genomic surveillance. Expert advice and epidemiological information provided by the NSW Health public health units are also gratefully acknowledged. The authors are indebted to all scientists and their organizations who have generously shared SARS-CoV-2 genome data on GISAID.

\section{Authors' contributions}

Data generation/collection/analysis was performed by AA, JD, RR, CL, RB and $J M$. Bioinformatic analyses and support was performed by RR, RS, MG and EM. $J D$ performed R coding. Drafting the manuscript was performed by AA, JD, $B M, J K, D D, S C$ and VS. The final manuscript was approved by all authors.

\section{Funding}

This study was supported by the Prevention Research Support Program funded by the NSW Ministry of Health and the National Health and Medical Research Council Centre for Research Excellence in Emerging Infectious Diseases [GNT1102962]. V.S. is supported by an Australian National Medical Research
Council fellowship [APP1123879]. The funders of this study had no role in study design, data collection, data analysis and interpretation, or writing of the article.

\section{Availability of data and materials}

The consensus genome sequences included in this study are all available from GISAID (www.gisaid.org). A full list of genomes and their corresponding IDs can be found in the Supplementary data (Additional file 1). The epifish package for $\mathrm{R}$, including a tutorial, is available from Github (https://github.com/learithe/epifi sh). The figure was produced using RStudio v1.2.5001-1.3.1056 and R v3.6.1, with the epifish package v1.0 [4], the fishplot package v0.5, [3], and the epifish dependencies "lubridate" (v1.7.4-1.7.9), "dplyr" (v0.8.3-1.0.2) and "tidyr" (v1.0.0) from the tidyverse [8].

\section{Declarations}

\section{Ethics approval and consent to participate}

Clinical specimens were routinely processed at the ICPMR and deemed not research. A non-research determination for this project was granted by Health Protection NSW since it was a designated communicable disease control activity.

\section{Consent for publication}

Not applicable.

\section{Competing interests}

The authors declare that they have no competing interests.

\section{Author details}

${ }^{1}$ Centre for Infectious Diseases and Microbiology Laboratory Services, NSW Health Pathology - Institute of Clinical Pathology and Medical Research, Westmead, NSW, Australia. ${ }^{2}$ Sydney Institute for Infectious Diseases, The University of Sydney, Sydney, NSW, Australia. ${ }^{3}$ Centre for Infectious Diseases and Microbiology - Public Health, Westmead Hospital, Sydney, NSW, Australia. ${ }^{4}$ Sydney Informatics Hub, Core Research Facilities, The University of Sydney, Sydney, NSW, Australia. ${ }^{5}$ Health Protection New South Wales, NSW Ministry of Health, Sydney, NSW, Australia. ${ }^{6}$ Children's Hospital at Westmead, Sydney, NSW, Australia.

Received: 29 April 2021 Accepted: 28 October 2021

Published online: 17 November 2021

\section{References}

1. Rockett RJ, Arnott A, Lam C, Sadsad R, Timms V, Gray KA, et al. Revealing COVID-19 transmission in Australia by SARS-CoV-2 genome sequencing and agent-based modelling. Nat Med. 2020;26(9):1398-404. https://doi.org/10. 1038/s41591-020-1000-7.

2. Eden J-S, Rockett RJ, Carter I, Rahman H, de Ligt J, Hadfield J, et al. An emergent clade of SARS-CoV-2 linked to returned travellers from Iran. Virus Evol. 2020. https://doi.org/10.1093/ve/veaa027.

3. Miller CA, McMichael J, Dang HX, Maher CA, Ding L, Ley TJ, et al. Visualizing tumor evolution with the fishplot package for R. BMC Genomics. 2016;17(1):880. https://doi.org/10.1186/s12864-016-3195-z.

4. Draper, J. Epifish package for R, https://github.com/learithe/epifish

5. McAnulty JM, Ward K. Suppressing the Epidemic in New South Wales. N Engl J Med. 2020;382: e74. https://doi.org/10.1056/NEJMc2011592.

6. Deng X, Gu W, Federman S, du Pleiss L, Pybus OG, Faria NR, et al. Genomic surveillance reveals multiple introductions of SARS-coV-2 into Northern California. Science. 2020;369(6503):682-7. https://doi.org/10.1126/science. abb9263.

7. Cyranoksi D. Alarming COVID variants show vital role of genomic surveillance. Nature. 2021;589:337-8. https://doi.org/10.1038/d41586-021-00065-4.

8. Wickham $\mathrm{H}$, et al. Welcome to the tidyverse. J Open Source Source Softw. 2021;4(43):1686. https://doi.org/10.21105/joss.01686.

\section{Publisher's Note}

Springer Nature remains neutral with regard to jurisdictional claims in published maps and institutional affiliations. 